\title{
Influence of consecutive sediment replenishment on channel bed morphology
}

\author{
L. Bösch \\ Federal Institute of Technology Zurich, ETHZ, Zurich, Switzerland \\ E. Battisacco, M.J. Franca \& A.J. Schleiss \\ École Polytechnique Fédérale de Lausanne, EPFL, Switzerland
}

\begin{abstract}
Dams interrupt the longitudinal connectivity of a river as they store water and trap sediment. When transport capacity downstream of dams exceeds sediment supply, water becomes hungry and channel incision, bed armouring and reduction of morphological diversity are the consequences. Sediment replenishment is an increasingly common measure to restore the sediment regime of such disturbed river reaches. In the present study the influence on channel bed morphology of different geometrical configuration of replenishments and of consecutive gravel augmentation is assessed by means of systematically laboratory experiments. The typical features of a straight armoured alpine gravel channel are reproduced on the model in terms of slope, cross section and bed grain size. The total amount of replenished sediment is deposited in four identical volumes following two different geometrical configurations (parallel and alternating). The reaction of the channel bed is analysed on image and laser data. Persistency of the first replenishment is high and it covers a large portion of the channel bed, which consequently decreases channel bed roughness remarkably. The persistency of the second replenishment is low and the effect on the depositional pattern established after the first replenishment only minor. This is explained by the smoothing of the channel bed during the first replenishment, resulting in higher transport capacities. For the alternating configuration an increase in the deposition heights in the very downstream channel reach can be observed due to the second replenishment. Thus it is assumed that consecutive replenishment can increase the impact length of restoration projects. The parallel configuration results in very spread depositions, while for the alternating configuration a clear bed form pattern could be observed with a wavelength of depositions corresponding to the length of the replenished volumes. These depositions are understood as mounds, forming the initial condition of alternating bars. Thus in order to enhance channel bed topography of an armoured layer downstream a dam, sediment replenishment added by the alternating configuration is most favourable and effective.
\end{abstract}

\section{INTRODUCTION}

Worldwide dams are built to provide water for irrigation, electricity and drinking. They represent one of the most grave human interventions in the hydrological cycle (McCartney, 2009). Regardless their purpose all dams store water and trap sediment, thus they interrupt the longitudinal river continuum and influence downstream flow and sediment regime (Kantoush et al. 2010a). If transport capacity of released water exceeds sediment supply (hungry water), bed erosion and coarsening of the bed surface (armouring) are the consequences (Kondolf 1997). This leads to habitat loss in the downstream river reach. For instance a loss in spawning habitats occurs, as fishes are not anymore able to move the large surface gavels to incubate their eggs (Kondolf 1997). Sediment supply is widely accepted to be one of the primary factor on morphology and gravel bar formation (Venditti et al. 2012) and a decrease in its contribution leads to a degradation of morphological complexity, as it was i.e. observed at Sacramento River in California (Kondolf 1997).

Today, revitalisation of disturbed river reaches forms an integral part of river management. For recreating and maintaining aquatic and riparian ecosystems, sediment transport and associated channel morphology are understood as key processes (Kantoush, et al. 2010a). Sediment replenishment is an increasingly common technique to mitigate the negative effects of sediment deficit (Ock et al. 2013). The principle of sediment replenishment is to excavate gravel from the reservoir and replenish it downstream of the dam into the river. By natural or artificial floods, replenished sediment 
is then returned to the river reach (Kantoush, et al. 2010a). Sediment replenishment projects aim to fine bed size material and to increase bed mobility and morphological complexity, such as rebuilding gravel bars (Sklar et al. 2009). Venditti et al. (2012) found by an experimental setup that bar loss due to sediment deficit is reversible by adding sufficient sediment. This highlights the main role of sediment supply in determining river morphology (Venditti et al. 2012). The success of river restoration by means of sediment replenishment depends on a variety of factors, such as discharge, grain size, volume and geometry of replenishment (Kantoush et al. 2010b). Ock et al. (2013) stated that all knowledge from past tests about sediment replenishment is not sufficient to draw general guidelines for river management downstream of dams. Especially the influence of discharge and sediment load on the river geometry and the timescales of changes due to sediment replenishment are essential to a better understanding of the physical processes inherent to gravel augmentation (Kantoush, Sumi \& Kubota 2010). Without more precise knowledge about these factors sediment replenishment cannot be adapted appropriately, which may result in undesirable ecological consequences or even in failure of the restoration project itself (Kantoush, Sumi \& Kubota 2010).

Volume of placed sediment defines how much and how long downstream river reach is influenced and how pronounced bed elevation and morphological changes may result. In addition, river restoration with regard to sediment deficit is a never-ending process, as long as upstream dams are not removed (Harvey et al. 2005). In several field studies, more than one sediment replenishment was undertaken to restore river reach (Bunte 2004). In the present study experiments with consecutive replenishment are performed. The main purpose of the study is to experimentally investigate the morphological responses on consecutive sediment augmentation in order to describe the effect of sediment replenishment on channel bed morphology, the persistency of formed depositions and if increased volume of sediment replenishments leads to enhanced variations in bed topography. Furthermore two different configurations are tested in order to evaluate the influence of initial geometrical placement on channel bed response.

\section{METHODS}

\subsection{Experimental setup}

The experiments were performed at the Laboratory of Hydraulic Constructions ( $\mathrm{LCH}$ ) at the École Polytechnique Fédérale de Lausanne (Bösch 2015).
The experimental channel at a scale of 1:10 represents a straight alpine gravel channel with a slope of $1.5 \%$, a length of $15 \mathrm{~m}$, a width of $0.4 \mathrm{~m}$ and a trapezoidal cross section (Fig. 1). The channel bed is fixed, thus accounting for bed armouring, and composed of gravels with a mean grain size diameter of $11.5 \mathrm{~mm}$ (see also Battisacco et al. 2015). Overall the experimental flume properties, such as slope, bed grain size and Froude number are representative for an alpine gravel channel (Parker et al. 2002, Hersberger 2002 \& Cui et al. 2003) and the applied scale of 1:10 can be considered as adequate for bed morphology modelling of mountain rivers (Weichert 2006 in Heller 2011).

The replenished sediment is added to the channel following the high-flow stockpile method (Ock et al. 2013) and coloured red in order to be distinguishable from the channel bed. $\mathrm{D}_{50}$ of replenished sediment is $5.5 \mathrm{~mm}$, thus smaller as bed grain sizes as it is suggested by Ock et al. (2013). Regarding the geometric scale factor, the used grain size distribution of the replenishment ranges from 30 to $80 \mathrm{~mm}$, which lies in the upper range of possible spawning gravels (Kondolf \& Wolman 1993). The replenishment is placed in four deposits, of equal shape and volume $\left(\approx 0.006 \mathrm{~m}^{3}\right)$ following two different configurations. In configuration $\mathrm{A}$ deposits are located parallel, in configuration B they are shifted by half a deposition length, resulting in an alternating character (Fig. 2). Discharge is provided and controlled by the recirculation system of the laboratory. Water and sediment are allowed to flow out freely at the downstream section.

Discharge was chosen such that bed shear stress was large enough to erode replenished sediment and small enough to let channel bed and banks immobile. In preliminary investigations optimal constant discharge was found at $100 \%$ of submergence of the replenishments, corresponding to a flow depth of $0.07 \mathrm{~m}$ and a discharge of $0.019 \mathrm{~m}^{3} / \mathrm{s}$. With this discharge effective erosion of the replenishments and largest area of impact was observed (Battisacco et al. 2015 \& Bösch 2015). The discharge was kept constant over the entire experiment. Initial hydraulic conditions in the experimental channel are listed in Table 1.

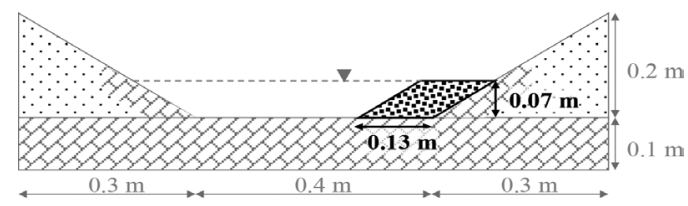

Figure 1. Schematic cross section of experimental channel with one replenishment volume (dark). 


\subsection{Experimental procedure}

To analyse the influence of consecutive sediment replenishment two different data sets are acquired: Image (2D) and Laser (3D-Topography). Image data are acquired with a GoPro3+-Camera, taking photos at 10 time steps for each replenishment experiment. Resolution of the images is $3 \times 3 \mathrm{~mm}^{2}$.

Channel bed elevations are measured by the laser (OADM 1317480/S35A, Baumer, Laser class 2) for initial and final condition of each replenishment experiment. The laser measurement accuracy is $\pm 1 \mathrm{~mm}$. To conduct the measurements the two instruments (Camera and Laser) are placed on a carriage, positioned at a fixed height, movable in downstream and cross sectional direction. Longitudinal laser profiles are taken at lateral position of $20 \mathrm{~mm}$ spacing, whereas longitudinal resolution is $1 \mathrm{~mm}$. In addition the placed and final washed out sediment was weighted for each replenishment experiment.

The total duration of the experiment is six hours. After placing the first replenishment into the channel, discharge is applied continuously over three hours. Then the tests are interrupted in order to undertake the laser measurements, to weight
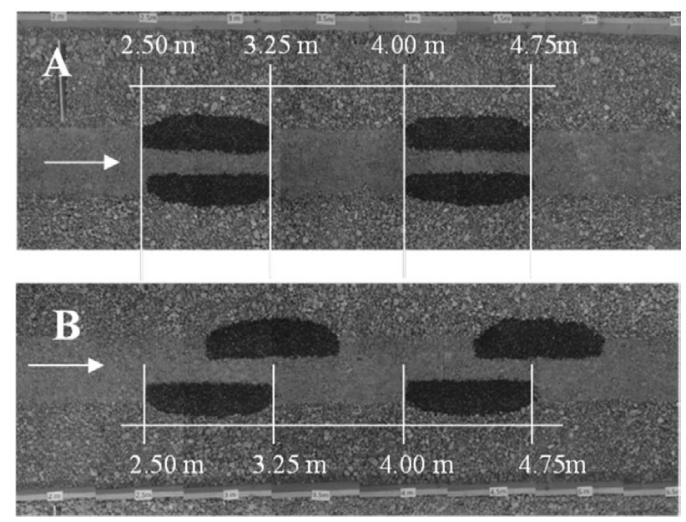

Figure 2. Experimental channel with replenished sediment (dark) for configuration A (top) and B (bottom). the washed out sediments and to refill the eroded replenishments to reach the same initial geometry as for the first replenishment. Then the tests are continued with the same discharge for another three hours. To avoid influence of the up- and downstream boundary conditions, the replenishments are deposited $2.5 \mathrm{~m}$ downstream of actual beginning of the channel, and data is only acquired down to $12.5 \mathrm{~m}$. This results in a final observation length of $10 \mathrm{~m}$.

\subsection{Data analysis}

The persistency of the sediment replenishment is defined as relation between weight of washed out and placed sediment.

To analyse the images, they were converted into binary images and the area of the channel bed was defined automatically (Bösch 2015). On the binary images the temporal evolution of the area covered by the replenishment is assessed. The Covered Surface (CS) is defined as relation between the area covered by the replenishment and the total channel bed area. The occupational rate along the channel (OCR) allows to assess the longitudinal distribution of the replenishment on the images. OCR is calculated as portion of covered channel bed at each cross section on the final images (after $3 \mathrm{~h}$ and $6 \mathrm{~h}$ )

The analysis of the laser data allows to assess the differences in channel bed elevation, the influence on bed roughness as well as the volumes of deposition after the repelnishments. The differences in channel bed elevation $\left(\Delta \mathrm{z}_{\mathrm{b}}\right)$ are obtained by subtracting the final height measurements from the initial ones. Thus positive values correspond to depositions of replenished sediment along the channel. The differences of each longitudinal profile are further averaged in cross sectional direction to be comparable with the OCR. Coleman et al. (2011) found standard deviation of bed elevations to provide a robust measure of bed roughness height. Thus to define how consecutive sediment replenishment influences channel bed roughness, standard deviation of bed elevation downstream of the replenishments are calculated before and

Table 1. Initial hydraulic conditions in the experimental channel.

\begin{tabular}{|c|c|c|c|c|c|c|c|c|}
\hline \multirow[b]{2}{*}{$\begin{array}{l}\text { Discharge } \\
{\left[\mathrm{m}^{3} / \mathrm{s}\right]}\end{array}$} & \multirow[b]{2}{*}{$\begin{array}{l}\text { Flow depth } \\
{[\mathrm{m}]}\end{array}$} & \multirow[b]{2}{*}{$\begin{array}{l}\text { Velocity } \\
{[\mathrm{m} / \mathrm{s}]}\end{array}$} & \multirow[b]{2}{*}{$\begin{array}{l}\text { Froude } \\
\text { number } \\
{[-]}\end{array}$} & \multirow[b]{2}{*}{$\begin{array}{l}\text { Flow } \\
\text { Reynolds } \\
\text { number }[-]\end{array}$} & \multicolumn{2}{|c|}{$\begin{array}{l}\text { Replenishment } \\
\left(D_{50}=5.5 \mathrm{~mm}\right)\end{array}$} & \multicolumn{2}{|c|}{$\begin{array}{l}\text { Channel bed } \\
\left(\mathrm{D}_{50}=11.5 \mathrm{~mm}\right)\end{array}$} \\
\hline & & & & & $\begin{array}{l}\text { Shields } \\
\text { shear } \\
\text { stress } \theta_{c}[-]\end{array}$ & $\begin{array}{l}\text { Grain } \\
\text { Reynolds } \\
\text { number }[-]\end{array}$ & $\begin{array}{l}\text { Shields shear } \\
\text { stress } \theta_{c}[-]\end{array}$ & $\begin{array}{l}\text { Grain } \\
\text { Reynolds } \\
\text { number [-] }\end{array}$ \\
\hline 0.019 & 0.07 & 0.54 & 0.65 & 34203.42 & 0.09 & 445.94 & 0.04 & 932.43 \\
\hline
\end{tabular}


after the replenishment. The standard deviations are calculated from the channel bed laser profiles, corrected by the channel slope. The volumes of deposition are calculated from the differences in bed elevation over the entire channel length. More precisely, the height differences are multiplied by the laser data resolution $\left(1 \times 20 \mathrm{~mm}^{2}\right.$, compare 2.2). To assess more in detail the influence of consecutive sediment replenishment on the channel bed morphology, the depositional pattern in the downstream channel reach is further analysed by looking at the Power Spectral Density (PSD) of the OCR and $\Delta z_{b}$. In many fields this is a common approach to reveal hidden periodicities in a signal (Stoica et al. 2005). The PSD is estimated only for the channel reach downstream of the replenishments, in order to avoid an influence on the signal by the original configuration. To only analyse the periodicity of fluctuations in the signal, the mean values of the signals (mean $\Delta z_{b}$ and final CS) are subtracted from the $\Delta z_{\mathrm{b}}$ and OCR. For the PSD, the entire downstream length (NFFT $=100 \%$ ) is used as data input. Window size is set to half of data length with an overlapping of $50 \%$, which is the recommended value for Welch's method, that was herein used (Stoica et al. 2005).

\section{RESULTS}

The persistency after the first replenishment is generally high. While for the alternating configuration B $96.6 \%$ of placed material stayed in the channel, for the parallel configuration A the persistency is smaller $(71.3 \%)$. The persistency of the second augmentation is significantly smaller than after the first replenishment (A: 13.9\%, B: 40.8\%). Thus not much of the secondly added material remains in the channel. This finding is further confirmed by the volumes of deposition, where increase due to second replenishment is only minor (Table 2). Nevertheless for the configuration B a relative increase in deposited volume by $20 \%$ is observed.

The temporal evolution of the Covered Surface (CS) shows that during the first replenishment after a rapid increase of $\mathrm{CS}$ at the beginning, an

Table 2. Volume of deposition along entire channel after 1 st $(3 \mathrm{~h})$ and 2 nd $(6 \mathrm{~h})$ replenishment.

\begin{tabular}{llll}
\hline & $\begin{array}{l}\text { Deposition } \\
3 \mathrm{~h}\left[\mathrm{~mm}^{3}\right]\end{array}$ & $\begin{array}{l}\text { Deposition } \\
6 \mathrm{~h}\left[\mathrm{~mm}^{3}\right]\end{array}$ & $\begin{array}{l}\text { Relative } \\
\text { increase* [\%] }\end{array}$ \\
\hline A & $9.24 \mathrm{E}+06$ & $9.60 \mathrm{E}+06$ & 4 \\
B & $1.10 \mathrm{E}+07$ & $1.31 \mathrm{E}+07$ & 20 \\
\hline
\end{tabular}

$*(2 \mathrm{nd}-1 \mathrm{st}) / 1 \mathrm{nd}$. equilibrium value is approach which lies around 0.38 for configuration $\mathrm{B}$ and 0.43 for configuration A (Fig. 3). The second replenishment had only a minor influence on the equilibrium value of $\mathrm{CS}$ for configuration A. In case of the alternating configuration the final CS is slightly increased to 0.43 nearly reaching the value for the parallel configuration (0.44).

For both configurations first replenishment leads to a remarkable bed fining, reducing initial standard deviation from around $\mathrm{D}_{50, \text { bed }}(11.5 \mathrm{~mm})$ to the $\mathrm{D}_{50 \text {,repl }}(5.5 \mathrm{~mm})$. The second replenishment slightly increased the bed roughness, while the effect is again more pronounced for the alternating configuration (Table 3).

In Figures 4 and 5 the distance along channel (x) is normalized by the channel width (w). Figure 4 shows the OCR and its cumulative sum for both configurations for first (solid line) and second replenishment (dashed line) over the channel reach downstream of the replenishments. From the first replenishment, clusters of high and low covered cross sections are established. For configuration B highest peak, with a cross section cover rate of more than 0.8 is observed at $\mathrm{x} / \mathrm{w} \approx 16$, thus around $1 \mathrm{~m}$ below the last replenishment. This initial maximal peak in the downstream OCR-signal is followed by other peaks, ranging between 0.6 and 0.7 . The analysis of the PSD shows one clear peak for configuration $\mathrm{B}$ at a wavelength of $1.5 \mathrm{w}$ (Fig. 6).

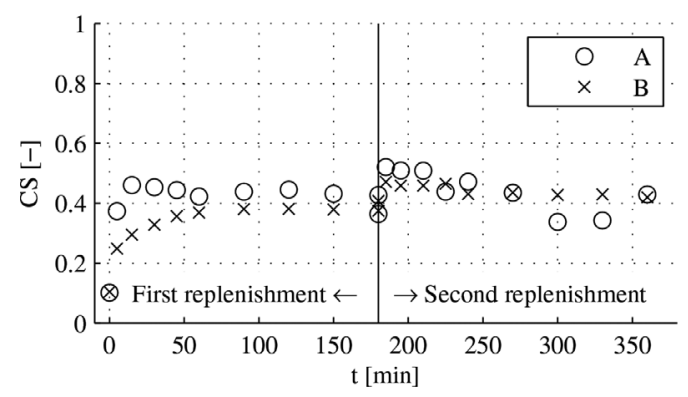

Figure 3. Temporal evolution of Covered Surface (CS) over the total duration of the experiment.

Table 3. Standard Deviation (STDV) along the channel reach downstream of the replenishments for initial condition, after 1 st $(3 \mathrm{~h})$ and after $2 \mathrm{nd}(6 \mathrm{~h})$ replenishment.

\begin{tabular}{lllll}
\hline & $\begin{array}{l}\text { STDV } \\
\text { initial }[\mathrm{mm}]\end{array}$ & $\begin{array}{l}\text { STDV } \\
3 \mathrm{~h}[\mathrm{~mm}]\end{array}$ & $\begin{array}{l}\text { STDV } \\
6 \mathrm{~h} \mathrm{[mm]}\end{array}$ & $\begin{array}{l}\text { Relative } \\
\text { increase* [\%] }\end{array}$ \\
\hline A & 11.09 & 5.31 & 5.49 & 3 \\
B & 12.59 & 6.89 & 7.24 & 5 \\
\hline
\end{tabular}

$*(2 \mathrm{nd}-1 \mathrm{st}) / 1 \mathrm{nd}$. 

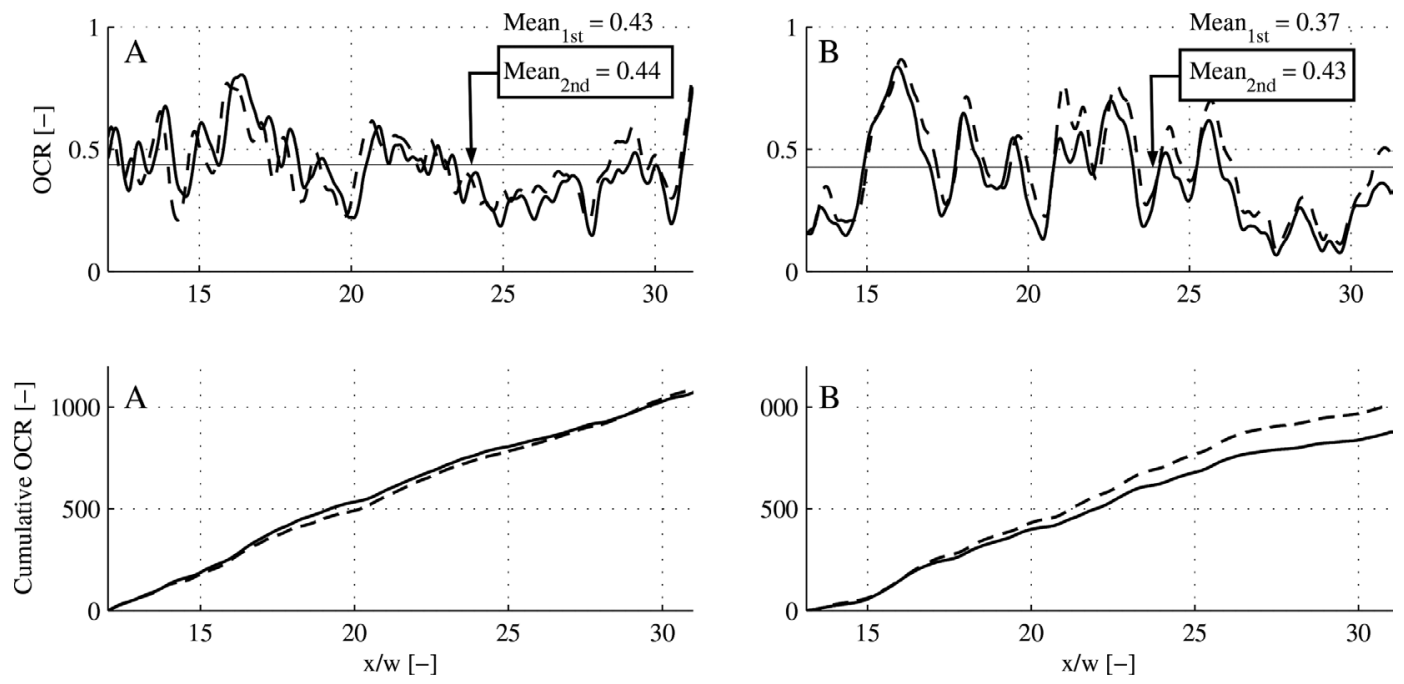

Figure 4. Occupational Rate (OCR) along channel reach downstream of the replenishments and their cumulative sum for configuration A (left) and B (right). First replenishment = solid line, second replenishment $=$ dashed line.
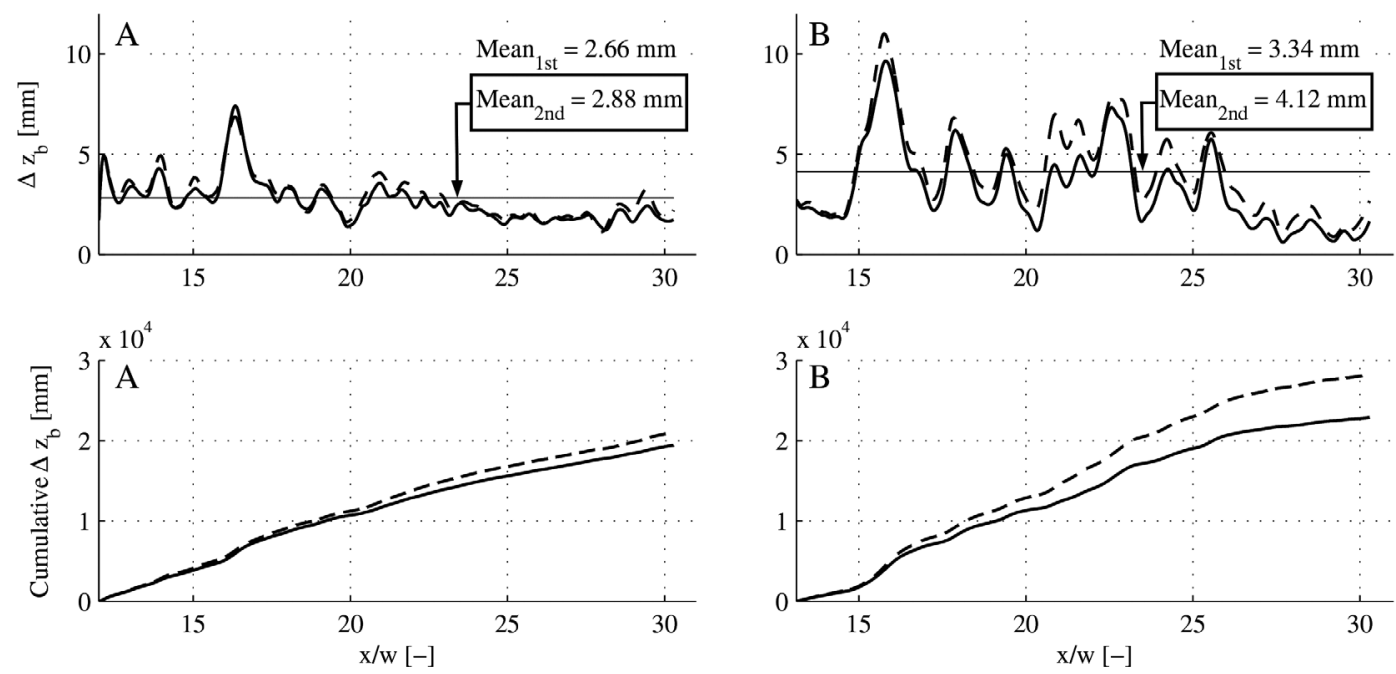

Figure 5. Elevation difference $\left(\Delta \mathrm{z}_{\mathrm{b}}\right)$ along channel reach downstream of the replenishments and their cumulative sum for configuration A (left) and B (right). First replenishment = solid line, second replenishment $=$ dashed line.

Thus the alternating configuration leads to periodic depositions along the downstream channel reach at a wavelength of $1.5 \mathrm{w}$. This result gets reasonable when looking at the pattern in OCR for configuration B: Indeed with a distance of less than $2 \mathrm{w}$, peaks in the OCR can be located visually. For the parallel configuration $\mathrm{A}$, the initial peak at $\mathrm{x} / \mathrm{w} \approx 16$ is also present. Further downstream no pronounced peaks in the OCR can be observed and consequently no peak is found in the PSD for this configuration. Thus, in contrast to the alternating configuration, the parallel one did not lead to periodic depositions along the downstream channel reach.

For both configurations the second replenishment does not change the spatial pattern of OCR established after the first replenishment: peaks and depletions are consistent. In case of the alternating configuration $\mathrm{B}$, the OCR seems to be increased by the second replenishment mainly 


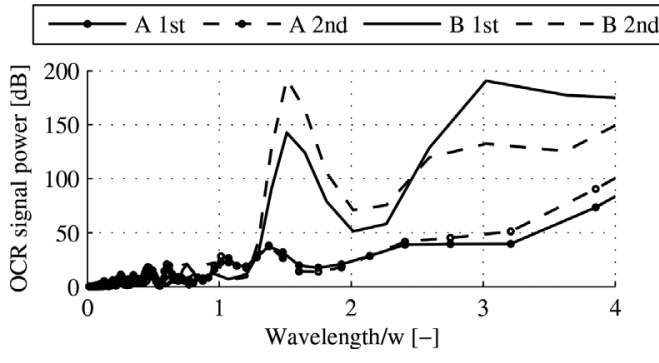

Figure 6. Power Spectral Density (PSD) of the OCR downstream of the replenishments.

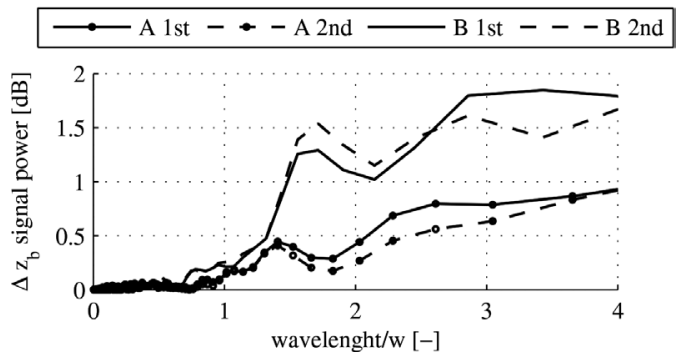

Figure 7. Power Spectral Density (PSD) of the $\Delta \mathrm{z}_{\mathrm{b}}$ downstream of the replenishments.

after $\mathrm{x} / \mathrm{w} \approx 17$. This observation is confirmed by the cumulative sum of OCR, where a difference between first and second replenishment can only be observed after this distance. For the parallel configuration no such trend can be identified, thus for this configuration the effect of the second replenishment is small over the entire channel length. Likewise the analysis of the PSD shows that the spatial pattern of OCR does not change due to second replenishment and that configuration $\mathrm{A}$ is affected less than configuration $\mathrm{B}$ (Fig. 6). For configuration B, peaks of high covered surface still occur at the same wavelength, but are increased in amplitude due to the second replenishment.

While after the first replenishment OCR shows periodic peaks of $140 \mathrm{~dB}(\approx 0.16 \mathrm{OCR})$ larger than the mean, after the second replenishment this difference is enlarged to $190 \mathrm{~dB}(\approx 0.25 \mathrm{OCR})$. For configuration A the PSD of OCR after the second replenishment does not differ from the PSD after the first gravel augmentation (Fig. 6).

Figure 5 shows the cross sectional averaged differences in bed elevation $\left(\Delta z_{b}\right)$ for both configurations for first (solid line) and second replenishment (dashed line) over the channel reach downstream of the replenishments. Elevation change in bed occurs only in positive direction (fixed bed), representing deposition of the replenished material along the channel. For configuration $\mathrm{B} \Delta \mathrm{z}_{\mathrm{b}}$ exceeds mean grain size diameter of replenishment $(5.5 \mathrm{~mm})$ at several locations, while no similar behaviour can be observed for configuration A. Correspondingly also mean deposition height is lower for configuration A $(2.66 \mathrm{~mm})$ than for configuration B $(3.34 \mathrm{~mm})$ after the first replenishment. As it was already observed in the PSD of the OCR, also the PSD of $\Delta z_{b}$ shows a more pronounced peak for configuration B (Fig. 7). From the PSD and the elevation differences along the channel it can be seen, that in case of configuration B periodically high depositions occur at a wavelength of around $1.7 \mathrm{w}$.

The influence of the second replenishment observed in the laser data $\left(\Delta z_{b}\right)$ corresponds to the image data (OCR). Generally peaks and depletions in the $\Delta z_{b}$ are consistent for the first and second replenishment, thus pattern established after the first augmentation remains. Nevertheless, in case of configuration B mean deposition height is remarkable increased to $4.12 \mathrm{~mm}$ and also peaks in depositions are generally higher, with a value of $1.33 \mathrm{~mm}(1.5 \mathrm{~dB})$ above the mean deposition height (Fig. 7). From the cumulative sum of $\Delta \mathrm{z}_{\mathrm{b}}$ for configuration B it can be seen that increase in deposition height due to the second replenishment mainly occurs after $\mathrm{x} / \mathrm{w} \approx 17$ as it was already the case for the OCR (Fig. 5). For the parallel configuration only a minor effect of the second replenishment can be observed: mean deposition height is only increased by $0.2 \mathrm{~mm}$ and no large difference in the cumulative sum can be observed (Fig. 5).

Furthermore the analysis of the laser and the image data leads to the assumption that the patterns of OCR and $\Delta z_{b}$ are highly correlated. This is further proved by analysing their (zero-lag) correlation coefficient (Bösch 2015). Indeed, correlation coefficient of 0.88 and 0.94 are determined between laser $\left(\Delta \mathrm{z}_{\mathrm{b}}\right)$ and image data (OCR) for configuration $\mathrm{A}$ and $\mathrm{B}$ respectively. This high agreement between laser and image data strengthens the soundness of the results and supports the method of monitoring effects of sediment replenishment by means of images.

\section{DISCUSSION}

The results after the first replenishment showed that persistency of the material is high and a large area of the channel bed is impacted by the replenishment. As replenishment grain size is smaller than the pre-existing bed, the large covered surface can explain the remarkable decrease in bed 
roughness due to the replenishment. Indeed, configuration $\mathrm{A}$, with the larger covered surface, also leaded to a wider smoothing than configuration $\mathrm{B}$. In general the present observation of bed fining is in agreement with the reaction of steep mountain gravel streams to sediment pulse input observed by Madej (2001) and with recent morphodynamic modelling, where a fining of the bed surface due to increased sediment supply is predicted (Ferguson et al. 2015).

This bed fining also explains the low persistency of the second replenishment and the consequently only minor effect on channel bed morphology. The decrease in roughness after the first replenishment results in higher shear stress, which leads to increased transport capacity as initial condition for the second replenishment. This explanation is strengthened by the results from Ferguson et al. (2015), where modelling of sediment supply showed that bed fining and consequently increased shear stress raised transport capacity by an order of magnitude. Thus in order to enhance the effect of a second replenishment, the applied discharges should be decreased to match the roughness conditions established after the first replenishment.

For the alternating configuration the development of periodic depositions, observed in the laser and image data, may indicate the initiation of a change in channel bed morphology. As it was already observed by Venditti et al. (2012) the periodic depositions can be understood as mounds, forming the initial condition of alternating bars. However the peaks in deposition height are not very pronounced. These minor height variations can be explained by the fixed channel bed in the experimental setup. This does not allow small replenished grains to filter into the armoured channel bed. It is known from literature that augmentation of fine grains and filtering of such into channel bed increases the mobilization of bed material (Venditti et al. 2010). Therefore it can be assumed that changes in channel bed topography and resulting deposition heights would be enhanced in case of a mobile bed. Additionally, unsteady flow and a wider range of replenished grain sizes might amplify elevation variations, as stated in Nelson et al. (2015).

The wavelength of the observed periodicities is with 1.5 to $1.7 \mathrm{w}$ close to the initial length of the replenishments $(0.75 \mathrm{~m})$. Thus it can be assumed that periodicity of depositions in the downstream channel reach after gravel augmentation is directly dependent on the length of the replenishments. This result is in agreement with the observations made by Venditti et al. (2012), where distance between bars was equal to their length. However, the obtained periodicity is way below the empirical founded periodicity of alternate bars of 6 to $10 \mathrm{w}$ (i.e. Ikeda 1983 in Whiting et al. 1993). As the observation length for the PSD analysis is only around $7 \mathrm{~m}$ it is not feasible to observe periodicities with a wavelength of more than $3.5 \mathrm{~m}(\approx 9 \mathrm{w})$. This might explain the differences between observed wavelength and empirically founded periodicities of alternate bars.

The secondly added material did not lead to a general change in the pattern of depositions along the downstream channel reach. Therefore it is assumed that the pattern established after the first replenishment is stable. Furthermore the only minor influence of the second replenishment indicates that volume of the first gravel augmentation was enough to create an equilibrium condition in the channel. Nevertheless, for the alternating configuration a slight increase in the covered surface is observed and more material is deposited along the channel after the second replenishment. The analysis of the cumulative OCR and $\Delta \mathrm{z}_{\mathrm{b}}$ shows that this increase is most likely to occur in the very downstream channel reach. Thus it can be assumed that for configuration B the amount of first replenishment was slightly too less to achieve an equilibrium over the entire channel reach and that a second replenishment could increase this equilibrium impact length. For the parallel configuration this balanced situation is already achieved after the first replenishment as no general increase in the OCR and $\Delta \mathrm{z}_{\mathrm{b}}$ signal can be detected after the second replenishment. It can be assumed that with increased transport capacity augmented sediment is transported further downstream, where the first replenishment had no impact and deposited there with a similar pattern as in the more upstream section. Thus consecutive replenishment seems to be a measure to increase the impact reach of sediment replenishment. Consequently only one point of replenishment implementation is needed. This is an advantage in case of limited access for sediment replenishment in the more downstream river reach and minimizes the disturbance of riparian habitats. Additionally by using only one upstream implementation point, the distance to the sediment excavation and consequently transport distance is reduced. Overall this can increase the feasibility of sediment replenishment and reduces the costs of restoration projects.

\section{CONCLUSION}

Sediment replenishment can follow different goals, such as the fining of armoured channel bed downstream a dam or the enhancement of channel bed elevation variations. In the present study different geometrical configuration of replenishments and 
the effects of consecutive gravel augmentation were experimentally investigated. The channel bed is influenced in different ways depending on the geometrical configuration. The alternated configuration is found to be more effective: persistency of material in the channel is higher and the development of periodic bed elevation changes can be observed only for this configuration. The periodic mounds, forming the initial condition for alternate bars, follow a wavelength which correspond to the length of replenishments. Although a remarkable bed fining is observed for both configurations, if this is the main goal of the river restoration, one may also think of applying configuration A instead of alternating configurations, as largest covered surface and corresponding bed fining is observed for this parallel configuration.

The decrease in roughness lead to higher transport capacities as initial condition for the second replenishment and consequently persistency and deposition volume of second replenishment is small compared to the first one. Therefore the second replenishment, thus an increase in replenishment volume, did not remarkably enhance elevation changes. It is assumed that channel bed variation can only be enhanced by either decreasing the discharge in order to match roughness conditions established after the first replenishment or by changing experimental conditions such as a mobile channel bed, unsteady flow and a wider range of grain size distribution.

The pattern of deposition established after the first replenishment is very stable. The volume of the first replenishment was enough to establish an equilibrium condition along the channel. In case of the alternating configuration this equilibrium condition was not reached over the entire channel length after first replenishment, and the second replenishment did remarkably influence the very downstream reach of the channel. Thus consecutive sediment replenishment is stated to be an effective measure in order to increase the impact length of river restoration projects. This is of essential importance in case of limited access for sediment replenishment along more downstream river reaches.

\section{ACKNOWLEDGEMENTS}

The research is financed by FOEN (Federal Office for the Environment, Switzerland).

\section{REFERENCES}

Battisacco, E., Maire, A., Franca, M.J. \& Schleiss, A.J., 2015. Physical modeling of artificial river replenishment techniques to restore morphological conditions downstream of dams. In Proceedings of the 36th IAHR World Congress, Delft.
Bösch, L., 2015. Consecutive sediment replenishment in alpine rivers: Quantitative assessment and reproducibility of morphological response, Lausanne: École Polytechnique Fédérale de Lausanne (Master thesis).

Bunte, K., 2004. Gravel Mitigation and Augmentation Below Hydroelectric Dams: A Geomorphological Perspective, Fort Collins.

Coleman, S.E., Nikora, V.I. \& Aberle, J., 2011. Interpretation of alluvial beds through bed-elevation distribution moments. Water Resources Research, 47(11), pp. 1-14.

Cui, Y., Parker G., Lisle T.E., Gott J., Hansler-Ball M.E., Pizzuto J.E., Allmendinger N.E. \& Reed J.M., 2003. Sediment pulses in mountain rivers: 1 . Experiments. Water Resources Research, 39(9), pp. 3/1-3/12.

Ferguson, R.I., Church, M., Rennie, C.D. \& Venditti, J.G., 2015. Reconstructing a sediment pulse: modeling the effect of placer mining on Fraser River, Canada. Journal of Geophysical Research: Earth Surface. Accepted article.

Harvey, B., McBain, S., Reiser, D., Rempel, L., Sklar, L.S. \& Lave, R., 2005. Key uncertainties in gravel augmentation: Geomorphological and biological research needs for effective river restoration, CALFED Science Program and Ecosystem Restoration Program Gravel Augmentation Panel report, Sacramento.

Heller, V., 2011. Scale effects in physical hydraulic engineering models. Journal of Hydraulic Research, 49(3), pp. 293-306.

Hersberger, D., 2002. Wall Roughness Effects on Flow and Scouring in Curved Channels with Gravel Bed, Lausanne: École Polytechnique Fédérale de Lausanne.

Ikeda, H., 1983. Experiments on bedload transport, bed forms, and sedimentary structures using fine gravel in the 4-meter-wide flume. Environmental Research Center papers, 2, pp. 1-78.

Kantoush, S., Sumi, T., Kubota, A. \& Suzuki, T., 2010a. Impacts of Sediment Replenishment Below Dams on Flow and Bed Morphology of River. In First International Conference, Coastal Zone management River Deltas and Low Land Coastlines. Alexandria.

Kantoush, S., Sumi, T. \& Kubota, A., 2010b. Geomorphic response of rivers below dams by sediment replenishment technique. In Proceedings of the River Flow 2010 Conference. Braunschweig, pp. 1155-1163.

Kondolf, G.M., 1997. Hungry Water: Effects of Dams and Gravel Mining on River Channels. Environmental Management, 21(4), pp. 533-551.

Kondolf, G.M. \& Wolman, M.G., 1993. The sizes of salmonid spawning gravels. Water Resources Research, 29(7), pp. 2275-2285.

Madej, M., 2001. Development of channel organization and roughness following sediment pulses in singlethread, gravel bed rivers. Water Resources Research, 37(8), pp. 2259-2272.

McCartney, M., 2009. Living with dams: Managing the environmental impacts. Water Policy, 11(1), pp. 121-139.

Nelson, P.A., Brew, A.K. \& Morgan, J.A., 2015. Morphodynamic response of a variable-width channel to changes in sediment supply. Water Resources Research, 51. 
Ock, G., Sumi, T. \& Takemon, Y., 2013. Sediment replenishment to downstream reaches below dams: implementation perspectives. Hydrological Research Letters, 7(3), pp. $54-59$.

Parker, G. \& Toro-Escobar, C.M., 2002. Equal mobility of gravel in streams: The remains of the day. Water Resources Research, 38(11), pp. 46-48.

Sklar, L.S., Fadde, J., Venditti, J.G., Nelson, P.A., Wydzga, M.A, Cui, Y. \& Dietrich, W.E., 2009. Translation and dispersion of sediment pulses in flume experiments simulating gravel augmentation below dams. Water Resources Research, 45(8), pp. 1-14.

Stoica, P. \& Moses, R., 2005. Spectral Analysis of Signals T. Robbins, ed., New Jersey: Prentice Hall.

Venditti, J.G., Nelson, P.A., Minear, J.T., Wooster, J. \& Dietrich, W.E., 2012. Alternate bar response to sediment supply termination. Journal of Geophysical Research: Earth Surface, 117(2), pp. 1-18.
Venditti, J.G. Dietrich, W.E., Nelson, P.A., Wydzga, M.A., Fadde, J. \& Sklar, L.S., 2010. Mobilization of coarse surface layers in gravelbedded rivers by finer gravel bed load. Water Resources Research, 46(7).

Weichert, R., 2006. Bed morphology and stability of steep open channels, Zurich: Swiss Federal Institute of Technology.

Whiting, J. \& Dietrich, E., 1993. Experimental constraints on bar migration through bends: Implications for meander wavelength selection. Water Resources Research, 29(4), pp. 1091-1102. 